\title{
Impact of Monthly Radioxenon Source Time-Resolution on Atmospheric Concentration Predictions
}

\author{
Michael Schöppner, ${ }^{1,2}$ Martin Kalinowski, ${ }^{3}$ Wolfango Plastino, ${ }^{1,2}$ Antonio Budano, ${ }^{2}$ Mario de Vincenzi, ${ }^{1,2}$ \\ Anders Ringbom, ${ }^{4}$ Federico Ruggieri, ${ }^{2}$ and Clemens Schlosser ${ }^{5}$
}

\begin{abstract}
The general characterisation of the global radioxenon background is of interest for the verification of the Comprehensive Nuclear-Test-Ban Treaty. Since the major background sources are only a few isotope production facilities, their source term has an emphasized influence on the worldwide monitoring process of radioxenon. In this work, two different datasets of source terms are applied through atmospheric transport modelling, to estimate the concentration at two radioxenon detection stations in Germany and Sweden. One dataset relies on estimated average annual emissions; the other includes monthly resolved measurements from an isotope production facility in Fleurus, Belgium. The quality of the estimations is then validated by comparing them to the radioxenon concentrations that have been sampled at two monitoring stations over the course of 1 year.
\end{abstract}

Key words: Medical isotope production facility, Radioxenon measurement, Environmental monitoring, Treaty verification, Comprehensive Nuclear-Test-Ban Treaty Organization (CTBTO).

\section{Introduction and Background}

Once entered into force, the Comprehensive Nuclear-Test-Ban Treaty (CTBT) prohibits its ratifying member states to conduct any kind of nuclear explosion within their control. The physical verification of the treaty obligations is based on three

1 Department of Physics, University of Roma Tre, Via della Vasca Navale 84, 00146 Rome, Italy. E-mail: schoeppner@fis.uniroma3.it

2 INFN, Section of Roma Tre, Via della Vasca Navale 84, 00146 Rome, Italy.

3 Carl Friedrich von Weizsäcker Center for Science and Peace Research (ZNF), Beim Schlump 83, 20144 Hamburg, Germany.

4 Swedish Defence Research Agency (FOI), Gullfossgatan 6, S-16490 Stockholm, Sweden.

5 Federal Office for Radiation Protection (BfS), Rosastraße 9, 79098 Freiburg, Germany. waveform technologies, i.e. seismic, hydroacoustic and infrasound, as well as radionuclide monitoring. For this purpose, the International Monitoring System (IMS) is built and already more than $80 \%$ operational. The noble gas component of the radionuclide monitoring is based on the emission of radioxenon during a nuclear explosion, which is transported through the atmosphere, even after being released from an underground explosion. A worldwide network of monitoring stations is constantly measuring the radioxenon concentration with daily sampling (Z̈̈HRINGER et al., 2009).

However, nuclear explosions are not the only sources of radioxenon. Nuclear power plants and isotope production facilities (IPF), e.g. for medical isotopes, have been recognized as the main background sources that could compromise the ability to detect nuclear weapon tests. When the location and emission strength of a source is known, the influence on the daily samples from the radionuclide monitoring stations can be simulated with atmospheric transport modelling (ATM). Therefore, the further characterization of the background source terms has been the aim of several works (KALINOWSKI and TUMA 2009; SAEY et al. 2010; WotaWA et al., 2010).

Isotope production facilities have been identified as the strongest emitters of radioxenon, with every IPF emitting about the same order of magnitude of radioxenon as all nuclear power plants together (SAEY, 2009). Therefore, the radioxenon source terms of the IPFs are of high interest for the understanding of the background signal. However, it cannot be taken for granted that the producing companies would publish the released quantities of radioxenon in near real time. Thus, estimations about their average yearly emissions have been made (KALINOWSKI et al., 2012). 
In this work, emission data from the National Institute for Radioelements (IRE) in Fleurus, Belgium, with a monthly resolution, is used to simulate the concentrations at the IMS radionuclide stations. The estimations over one year are compared to the sampled concentration data of two IMS radionuclide stations. These results, based on the more accurate source terms, are then compared to the concentrations based on the previously estimated average yearly emissions.

\section{Materials and Methods}

\subsection{Atmospheric Transport Modelling}

When particles are emitted in a certain time interval from a given point on the globe, they can be transported through the atmosphere. Thus, they can arrive at a certain different location at a later point in time in a diluted concentration. For this matter, ATM can be used to estimate the time-dependent relation between two locations on a global (or regional) grid. These two points shall be called source and receptor; and the relation between them, source-receptorsensitivity (SRS). If the emission of the source or the diluted concentration at the receptor is known, the other one can be estimated with the SRS value as calculated by ATM. Of course, the results strongly depend on the meteorological conditions of the regarded time period, as well as on local atmospheric patterns that are not resolved by the simulation, which can lead to altered signals (PLAstino et al., 2010). Particles, which have been emitted in one time interval, can contribute at different arrival times to the concentration at the receptor site, via their various trajectories through the atmosphere.

The relation between a source and a receptor can be described with a source-receptor sensitivity matrix. The concentration $c\left(\mathrm{~Bq} / \mathrm{m}^{3}\right)$ at any given receptor can be expressed as the product of a spatiotemporal source field $S(\mathrm{~Bq})$ and a corresponding source-receptor sensitivity field $M\left(\mathrm{~m}^{-3}\right)$ at discrete locations $(i, j)$ and time intervals $n$ :

$$
c=M_{i j n} \cdot S_{i j n} .
$$

The field $S$ is a multidimensional array of sources, which is transformed by the multidimensional array of multiplicators $M$ into the concentration $c$ that is measured at the receptor (WотAWA et al., 2003). Here $M$ presents the sensitivity between source and receptor and has the dimension of $\mathrm{m}^{-3}$, whereas the inverse element of $M$ can be depicted as a dilution volume. However, while the underlying calculations are naturally three-dimensional in space, the produced SRS matrix $M$ is only two-dimensional, with time as a third dimension. General ATM software can simulate the transport of particles released from point, line, area or volume sources. The simulations can include long-range and mesoscale transport, diffusion, and deposition, as well as radioactive decay, into the calculations. In ATM, it is usually distinguished between forward and backward modelling, where both methods have advantages and disadvantages. Forward modelling is more efficient when the number of known sources is limited and the receptors are undefined. Backward modelling is more efficient when the number of receptors is limited and the sources are numerous or unknown. Therefore, in the case of IMS radionuclide sampling, the backward mode is preferred, as the location of the receptor site is well known.

\subsection{Data Status}

The concentration at IMS radionuclide stations can be estimated with ATM and the use of an emission database. In this case, such a database contains information about the location and strength of all contemplated sources with regard to ${ }^{133} \mathrm{Xe}$, i.e. 200 nuclear power plant sites (KaLINOwSKI, Tuma, 2009), and five IPF are considered. The emissions of nuclear power plants are estimated to be in the orders of $1 \mathrm{E}+10$ and $1 \mathrm{E}+13 \mathrm{~Bq}$ per year. Usually, emissions from NPPs are batch-released and below the boundary of semi-continuous regime (KALINOWSKI, Tuma, 2009), but since the detailed, time-resolved emission data is generally unknown, only continuous emissions can be used in the simulation. The impact of batch emissions is lower for NPPs away from the detector than for NPPs closer to the detector. On the other hand, isotope production facilities are rarer, but they usually produce a higher output of radioxenon, i.e. orders of $1 \mathrm{E}+13$ and $1 \mathrm{E}+16 \mathrm{~Bq}$ per year are estimated. Three of the five IPF are in the northern hemisphere and typically 
influence the radioxenon measurements in Europe, namely the ones in Fleurus (Belgium), Chalk River (Canada) and Petten (Netherlands); the latter usually emits radioxenon in the order of magnitude of average NPPs. Each known source is allocated to the nearest grid point as resolved by the ATM, i.e. in this case $1^{\circ} \times 1^{\circ}$. One database (a) handles all these sources as constant emitters of radioxenon, based on commonly accepted estimations of their average yearly emission. The second database (b) is similar with the exception that the IPF in Fleurus, Belgium, is handled as a varying source, based on reported emission data with monthly resolution from 2008. As these data are confidential, they are not published here, but are only described quantitatively. The year 2008 is characterized by the first five months with average emission strength, then a five months period during summer/autumn with emission particularly below the average, and towards the end of the year, two months with emissions clearly above average. The overall reported emissions are slightly lower than the usually applied estimation of $10^{15} \mathrm{~Bq}$ per year. Thus, the total radioxenon emission inventory for the year 2008 is lower in dataset (b) than in dataset (a).

To compare the effect of these two datasets on ATM-based radioxenon estimations, two IMS radionuclide stations have been selected; the German DEX33 at Schauinsland Mountain close to Freiburg, and the Swedish SEX63 close to Stockholm. With regard to ${ }^{133} \mathrm{Xe}$, a total of $19724 \mathrm{~h}$-samples are available from the German station, resulting in a total time coverage of $54 \%$ for the calendar year 2008 . From the Swedish station, 581 12-h samples are available, resulting in a total time coverage of $79 \%$ for the calendar year 2008. These samples also include non-detections of radioxenon, i.e. the concentrations are below the minimum detectable concentration (MDC), which has to be at $1 \mathrm{mBq} / \mathrm{m}^{3}$ or lower, by design criteria (SAEY, SCHLOSSER et al., 2010). However, after being reviewed from the International Data Centre (IDC) of the CTBTO the data is still subject to uncertainties due to the complex detection process (AUER et al., 2010). For each of these 779 samples, a Flexpart simulation has been conducted to produce the accordant SRS fields.

The meteorological fields are provided from the European Centre for Medium-Range Weather
Forecasts (ECMWF), and have spatial resolution of $1^{\circ}$ in latitude/longitude and a time resolution of $3 \mathrm{~h}$. Thus, the calculated SRS fields also have the same resolution in space and time.

In terms of estimated source terms, other relevant isotopes and isomers of xenon (namely ${ }^{131 \mathrm{~m}} \mathrm{Xe}$, ${ }^{133 \mathrm{~m}} \mathrm{Xe}$ and ${ }^{135} \mathrm{Xe}$ ) could be included, but the accordant data basis of IMS samples is not sufficient to cover a significant part of 2008. However, the emissions of these radionuclides are included in the simulation-particularly to simulate the feeding of ${ }^{133}$ Xe through ${ }^{133 m} \mathrm{Xe}$.

\section{Analysis and Discussion}

The two radioxenon emission databases have been applied on the calculated SRS fields, to determine the estimated radioxenon concentrations at the two selected stations. The simulated concentration values for the German (Swedish) station are presented and compared to the measurements in Fig. 1 (Fig. 2); the 197 samples (581 samples) are chronologically ordered with regard to their date and time of sampling. This means that the horizontal axis does not fully reflect the calendar year 2008, but just the order in which the samples were taken. The horizontal axis crosses the vertical axis at $1 \mathrm{e}-03 \mathrm{~Bq} / \mathrm{m}^{3}$, which is the official MDC for IMS radioxenon sampling (SAEY, SCHLOSSER et al., 2010).

As seen, for both stations, both simulations depict the characteristics of the time series rather well, including many maxima and minima; only for some time periods, the signal is off, or e.g. even predicts a minimum, where in reality there is a maximum (or vice versa). However, it has to be clearly stated that both kinds of simulation produce results of nearly the same quality. The statistical covariance and correlation have been calculated for each simulated time series, with regard to the experimental data. Table 1 shows that they are either similar for the simulations based on the different datasets, or even better for the simulation that is based on the all-constant dataset (a). The reason for this will become clear, when looking closer at the composition of the samples, cp. below. Nevertheless, due to the regional density of legitimate radioxenon emitters, each with emissions 


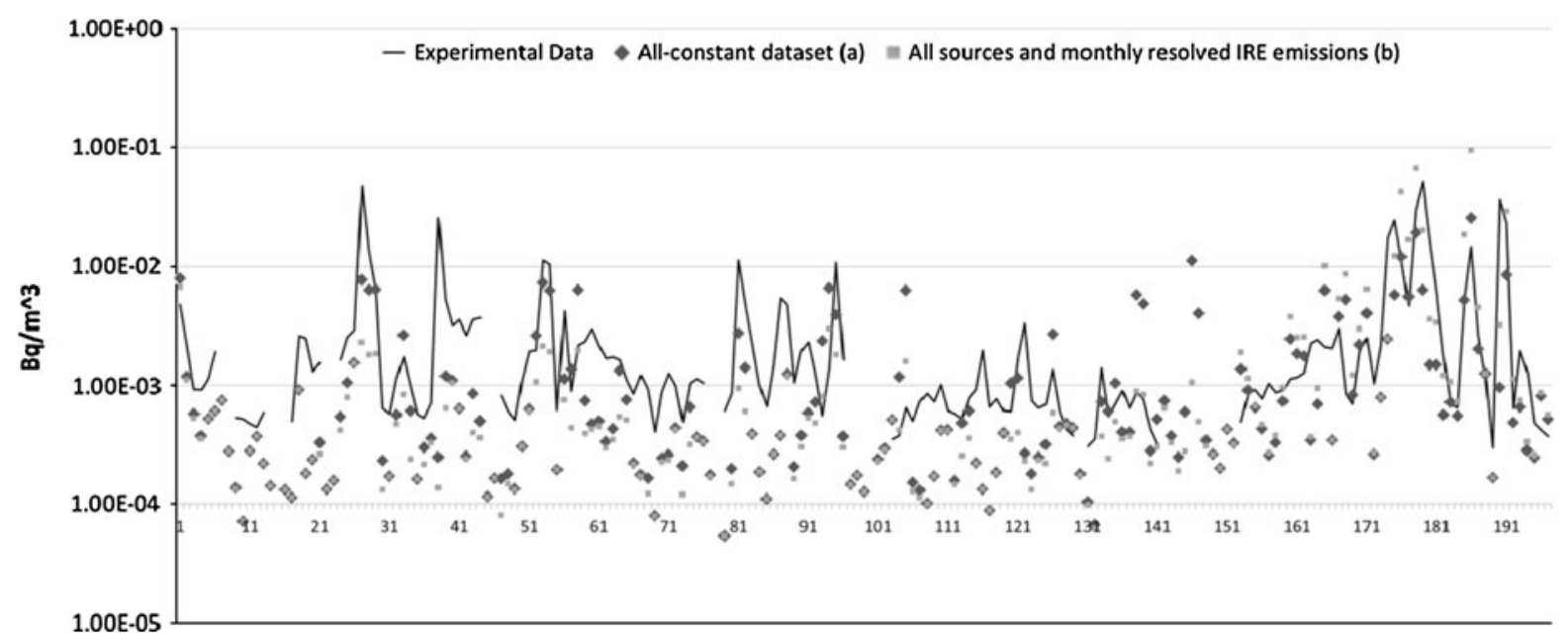

Figure 1

Comparison of the simulations based on two radioxenon emission datasets with the experimental data for the German IMS station DEX33

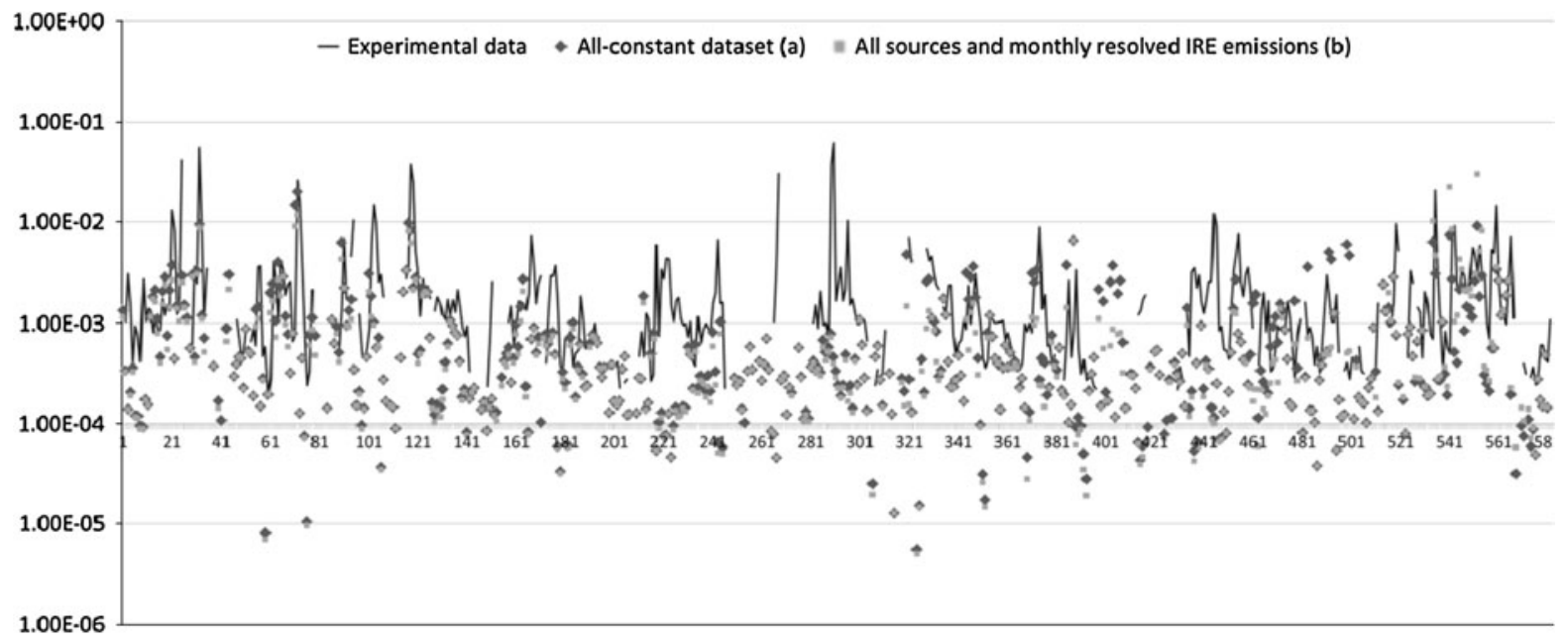

Figure 2

Comparison of the simulations based on two radioxenon emission datasets with the experimental data for the Swedish IMS station SEX63

Table 1

Statistical parameters of simulations for the German and the Swedish station based on datasets (a) and (b)

\begin{tabular}{llllll}
\hline & \multicolumn{2}{c}{ Covariance $\sigma$} & & \multicolumn{2}{c}{ Correlation $\rho$} \\
\cline { 2 - 3 } \cline { 5 - 6 } & Dataset (a) & Dataset (b) & & Dataset (a) & Dataset (b) \\
\hline DEX33 & $1.13 \mathrm{E}-05$ & $3.06 \mathrm{E}-05$ & & 0.50 & 0.42 \\
SEX63 & $4.01 \mathrm{E}-06$ & $3.08 \mathrm{E}-06$ & & 0.40 & 0.30 \\
\hline
\end{tabular}

that can only be estimated, in none of the presented scenarios does the correlation come close to the ideal value of one.

\subsection{Total Yearly Concentration}

Before going into the statistical analysis of the data, it is obvious that the simulated concentration values are, over the course of one year, rather too low than too high. When all collected samples available from 2008 are summed, they accumulate to $0.58 \mathrm{~Bq} / \mathrm{m}^{3}$ for the German station, while the simulation produces only $0.30 \mathrm{~Bq} / \mathrm{m}^{3}$ with the all-constant sources dataset (a), and $0.45 \mathrm{~Bq} / \mathrm{m}^{3}$ with the dataset (b), including the IRE time resolved emissions. The situation is similar for the Swedish station; while the collected samples add up to a total of $1.12 \mathrm{~Bq} / \mathrm{m}^{3}$, the simulations only produce 
$0.47 \mathrm{~Bq} / \mathrm{m}^{3}$ and $0.42 \mathrm{~Bq} / \mathrm{m}^{3}$, respectively. This is most likely due to a generally underestimated global radioxenon emission inventory.

However, within the simulated radioxenon signals, the contributions from IRE, Belgium, to the total concentration of 2008, are as follows: At the German station for dataset (a) $0.22 \mathrm{~Bq} / \mathrm{m}^{3}$ (of the aforementioned total of $0.30 \mathrm{~Bq} / \mathrm{m}^{3}$ ) and for dataset (b) $0.38 \mathrm{~Bq} / \mathrm{m}^{3}$ (of $0.45 \mathrm{~Bq} / \mathrm{m}^{3}$ ) are accounted due to IRE. At the Swedish station for dataset (a) $0.23 \mathrm{~Bq} / \mathrm{m}^{3}$ (of $0.47 \mathrm{~Bq} / \mathrm{m}^{3}$ ) and for dataset (b) $0.19 \mathrm{~Bq} / \mathrm{m}^{3}$ (of $0.42 \mathrm{~Bq} / \mathrm{m}^{3}$ ) are accounted due to IRE.

Though the IPF in Fleurus, Belgium, is usually the strongest regional emitter of radioxenon in Europe, supra-regional sources also contribute to the signals at the German and the Swedish stations, and have to be taken into account. During the year 2008, the IPF in Chalk River, Canada, regularly contributed to the concentrations at both stations: The estimated constant source term of $10^{16} \mathrm{~Bq} / \mathrm{m}^{3}$ contributes with $0.04 \mathrm{~Bq} / \mathrm{m}^{3}$ to the German station and $0.14 \mathrm{~Bq} / \mathrm{m}^{3}$ to the Swedish station, over the total period of 2008 . Compared to the total impact from IRE, this means that the IPF in Chalk River, Canada, plays usually only a minor role in the daily samples of the German station, but can significantly contribute to the daily samples of the Swedish station.

\subsection{Quality of Simulations of Single Samples}

In order to validate the quality of the simulations for single samples, two values have been calculated for each simulated sample: (1) The fraction of the IRE-contribution to the total sample concentration, and (2) the ratio of the simulated total sample concentration to the accordant experimental value. This means that (1) can vary between 0 and 1 , depending on how much of the simulated concentration of one sample results from IRE emissions; and (2) can take any positive value, but ideally should be 1 , since it is simply the ratio of simulation to experimental data.

Both values are then put in one diagram, and are sorted in ascending order with regard to the share of the IRE-contribution to the total simulated concentration (1). This means the samples with the lowest fractions of IRE-contributions are seen in the left end of the diagram, while the samples with the highest fractions are on the right end of the diagram; i.e. the series is monotonically increasing with regard to (1). Of course, the ratio of simulation to experimental data (2) stays attached to each sample.

The results for the German station are shown in Fig. 3 for the simulation based on dataset (a) and (b). As seen, there is a correlation between (1) and (2); for samples with a high share of IRE-contribution, the ratio between simulated and experimental data is wider spread, but on average closer to one. This signifies that, when, due to atmospheric conditions and/or a high emission, the share of the IREcontribution to the simulated sample concentration is high, and the simulation of the total concentration is more likely to reflect the experimental data (i.e. on average closer to 1). The smaller spread on the left end results from the more steady contributions from nuclear power plants, which can act as an area source, while the larger spread on the right end results from an insufficient time resolution of the IRE source term.

For the Swedish station, the results are presented in the same manner in Fig. 4. Here, the correlation between (1) and (2) is not as noticeable as it is for the German station. A slight convergence of (2) towards 1 can be observed for high values of (1). However, the effect is more prominent for the simulation based on the all-constant sources dataset (a). The fact that the correlation between IRE-contribution and the quality of the simulation is weaker than for the German station is most likely due to the fact that for the Swedish station, the IPF in Chalk River, Canada, also plays a comparably important role. Therefore, samples from the Swedish station can have up to two major contributors, IRE and CLK, and are thus less dependent on a single one of them.

\section{Conclusion and Outlook}

It has been shown that, although the simulations can depict quite well the general characteristics of the experimental signal, the dataset based on time-resolved source terms did not improve the quality of the simulation. This is equally valid for both the German and the Swedish station. Compared to the previously used estimations of annual total emissions, the reported 

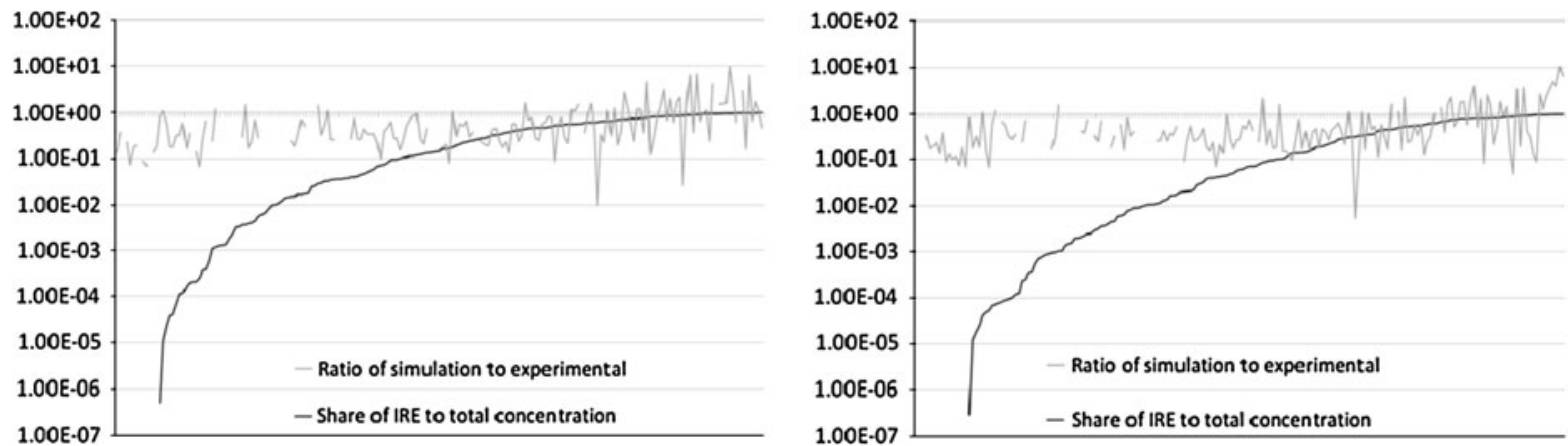

Figure 3

The effect of the share of IRE of the total concentration on the quality of the simulation for the German station; left the simulation for the allconstant dataset (a), right the simulation for the monthly resolved IRE dataset (b)

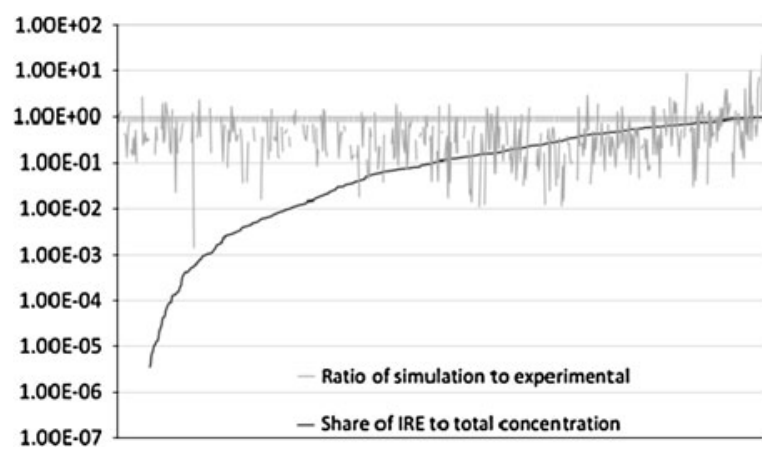

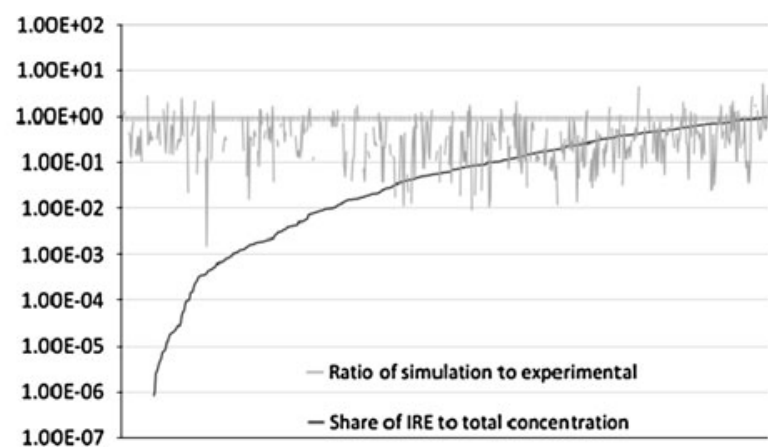

Figure 4

The effect of the share of IRE of the total concentration on the quality of the simulation for the Swedish station; left the simulation for the allconstant dataset (a), right the simulation for the monthly resolved IRE dataset (b)

monthly emission data can produce better results only in some cases. Generally, the previously used dataset (a) leads to a simulated time series of statistically higher quality. This is most likely due to the fact that the global radioxenon emission inventory is generally underestimated and dataset (b) is built on an even lower radioxenon emission inventory resulting from the IRE emission reports.

The reasons for a general underestimation of the radioxenon detections can be manifold, i.e. be found within the atmospheric transport model; additional/ unknown sources of radioxenon exist; or the real source terms are higher than used in the simulation. The model used here, Flexpart, has been proved in the analysis of tracer experiments not to produce particular over-estimations or under-estimations, when emissions and detections are well known (Sтонl et al., 1998). Since simulated samples for the German station with high contributions from IRE are more likely to reflect the experimental data, it is believed that the underestimation refers to the collective of nuclear power plants found in Western Europe, rather than to IRE itself. This conclusion cannot be drawn from the simulations for the Swedish station, because here the situation is more complicated, involving IRE and CLK in equal measure.

However, the presented results suggest that a time resolution higher than monthly is necessary, e.g. daily emission values from major radioxenon emitters, in order to significantly improve the quality of the ATM-based simulation for radioxenon concentrations in the future.

\section{Acknowledgments}

The authors highly acknowledge the support by the National Scientific Committee Technology of INFN 
for the ERMES project, and the European Commission under the FP7 programme for the EUMEDGRID project (Grant RI-246589). The authors are grateful to IRE, Belgium, for the provision of data, specifically Benoit Deconninck, and to the Grid Lab of INFN and the Department of Physics of University of Roma Tre, specifically to Federico Bitelli.

\section{REFERENCES}

Auer, M., Kumberg, T., Sartorius, H., Wernsperger, B., SchlosSER, C. (2010), Ten years of development of equipment for measurement of atmospheric radioactive xenon for the verification of the CTBT, Pure Appl. Geophys. 167, 471-486.

Kalinowski, M.B., and Tuma, M.P. (2009), Global radioxenon emission inventory based on nuclear power reactor reports, Journal of Environmental Radioactivity 100, 58-70.

Kalinowski, M.B., Grosch, M., Hebel, S. (2012), Assessment of radioxenon emissions from medical isotope production based on worldwide technetium-99 $\mathrm{m}$ consumption, Pageoph Topical Volume II.

Plastino, W., Plenteda, R., Azzari, G., Becker, A., Saey, P.R.J., Wotawa, G. (2010), Radioxenon time series and meteorological pattern analysis for CTBT event categorisation. Pure and Applied Geophysics 167, 559-573.
SAEY, P. (2009), The influence of radiopharmaceutical isotope production on the global radioxenon background, Journal of Environmental Radioactivity 100, 396-406.

SAey, P., Bowyer, T., Ringbom, A. (2010), Isotopic noble gas signatures released from medical isotope production facilitiesSimulations and measurements, Applied Radiation and Isotopes 68, 1846-1854.

SAEy, P., Schlosser, C., et al. (2010), Environmental Radioxenon Levels in Europe: a Comprehensive Overview, Pure and Applied Geophysics. doi:10.1007/s00024-009-0034-z.

Stohl, A., Hittenberger, M., Wotawa, G. (1998), Validation of the Lagrangian particle dispersion model Flexpart against largescale tracer experiment data, Atmospheric Environment Vol. 32 No. 24 , pp. $4245-4264$

Wotawa, G., et al. (2003), Atmospheric transport modelling in support of CTBT verification-overview and basic concepts, Atmospheric Environment 37, 2529-2537.

Wotawa, G., Becker, A., Kalinowski, M.B., Saey,P., Tuma, M., ZäHRINGER, M. (2010), Computation and Analysis of the Global Distribution of the Radioxenon Isotope 133-Xe based on Emissions from Nuclear Power Plants and Radioisotope Production Facilities and its Relevance for the Verification of the NuclearTest-Ban Treaty, Pure Appl. Geophys. 167, 541-557.

Zähringer, M., Becker, A., Nikkinen, M., Saey, P., Wotawa, G. (2009), CTBT radioxenon monitoring for verification: today's challenges, J Radioanal Nucl Chem 282, 737-742.

(Received December 1, 2011, revised May 4, 2012, accepted May 5, 2012) 